\title{
¿LOS MIGRANTES PERUANOS CONTRIBUYEN A LAS EXPORTACIONES DEL PAÍS?
}

\section{DO PERUVIAN MIGRANTS CONTRIBUTE TO THE COUNTRY'S EXPORTS?}

\section{Julio Alberto Hennings Otoya*}

Docente Auxiliar de la Facultad de Ciencias Contables, UNMSM

[Recepción: Abril de 2010 / Conformidad: Mayo de 2010]

Un viajero sabio nunca desprecia su propio país

Carlo Goldoni

\section{RESUMEN}

El presente artículo busca responder a la interrogante si los migrantes peruanos, cuyo número estaría superando al $10 \%$ de la población que vive en el suelo patrio, viene contribuyendo de alguna forma con el desarrollo de las exportaciones nacionales, considerando que conforman un segmento de consumidores con características propias, costumbres y preferencias vinculadas a su tierra natal.

El hecho anterior, aunado a factores como un mejor poder adquisitivo de los que han emigrado a países desarrollados, la variedad de recursos nacionales considerados de origen o "étnicos", la fama y el auge que está adquiriendo la cocina peruana entre otros atractivos nacionales podrían estar generando una oportunidad de mercado para muchas entidades productivas, comerciales $y$ turísticas del país.

Luego de un proceso de análisis de estudios referidos al tema, confrontados con la realidad aplicada en nuestro medio, se generan las conclusiones y recomendaciones, que pudieran ser aprovechadas por empresas peruanas que busquen desarrollar su potencial en el mercado internacional, haciéndose también hincapié que la profundización del

\begin{abstract}
This article seeks to answer the question whether Peruvian migrants, whose number would exceed $10 \%$ of the population living in the homeland, has contributed in some way with the development of national exports, considering that comprise a consumer segment with its own characteristics, habits and preferences related to their homeland.

The last fact, combined with factors such as improved purchasing power of those who have emigrated to developed countries, the variety of resources considered national origin or "ethnic" fame and the boom that is taking the peruvian cuisine and other national attractions would be generating a market opportunity for many entities in production, trade and tourism in the country.

After a process of analysis of studies relating to the subject, confronted with the reality applied in our environment, are generated the conclusions and recommendations, which could be exploited by $\mathrm{Pe}$ ruvian companies seeking to develop their potential in the international market, making it also stressed that the deepening of the subject could provide a framework for policies and actions of State, to integrate
\end{abstract}

* Economista, UNMSM. Docente Auxiliar de la Facultad de Ciencias Contables, UNMSM. 
tema podría servir de marco para establecer políticas y acciones de Estado, para integrar a los emigrantes peruanos en las estrategias de desarrollo del Perú.

Palabras clave: Migración internacional, globalización, remesas, productos "étnicos".

\section{INTRODUCCIÓN}

El Perú, a pesar de los éxitos macroeconómicos de los últimos años, continúa como mudo testigo del éxodo de numerosos ciudadanos hacía diversos países, movilidad justificada mayormente por expectativas laborales o por la búsqueda de una mejor educación. Este problema algo soslayado en las políticas de Estado, en realidad es parte de un fenómeno mayor de nuestros días, la migración internacional, -que si bien no es inédito en la historia- está agudizándose con el proceso de globalización que vivimos, donde se integran cada vez más las economías, el comercio y el intensivo flujo de capital que no conoce fronteras y que se potencian con el avance incesante de las tecnologías de información y comunicaciones (TIC's).

Si meditamos el problema desde un ángulo socio-económico, el fenómeno de la migración internacional nos mostrará costos y beneficios, tanto para las personas como para las naciones involucradas. Estos costos y beneficios se dan en los países que envían trabajadores como en los que lo reciben ${ }^{1}$, los primeros reiteradamente vinculados a la pérdida de capital humano (Brian Drain) y los segundos, mayormente a las remesas recepcionadas; sin embargo, al lado de estos sub-
Peruvian migrants in Peru's development strategies.

Key words: International migration, globalization, remittances, products "ethnic". yacen otros temas importantes de impacto social, comercial, cultural, etc. que concurren con el acontecimiento de la migración en sí.

En el presente artículo -sin ánimo de expresar prevalencia sobre dichos factores-, focalizaré el tema en los beneficios que generan los migrantes, tomando sus contribuciones (conscientes o no) a las exportaciones de nuestro país, generando con ellas oportunidades de desarrollo a las entidades productivas y comerciales de nuestro medio.

\section{Antecedentes teóricos}

Según la Organización de las Naciones Unidas, "migrante internacional es cualquier persona que cambia su país de residencia usual, es decir, el país donde vive. Los viajes temporales de recreación, vacaciones, negocios, tratamiento médico o peregrinación no son tomados como un cambio en el país de residencia" 2 . Otra mención precisa es la definición que hace George Borjas sobre la migración internacional, cuando manifiesta que es el "movimiento de personas o familias a través de las fronteras nacionales"

Entre las causales de las migraciones internacionales, ya en el siglo XIX Ravenstein ${ }^{4}$ señalaba que los flujos migratorios eran ge-

1 Aquino Rodríguez, Carlos (2005). Migración de peruanos a Japón y oportunidades de negocios. Web: www.geocities. com/Eureka/Plaza/1406

2 Diccionario de Términos de la Base de Datos de la ONU. En: http://unstats.un.org/unsd/cdb/cdb

3 Borjas, G. (2000). "Economics of migration”. International Encyclopedia of the Social and Behavioral Sciences.

4 Ravenstein, E. (1885 y 1889). “The laws of migration”. Journal of the Royal Statistical Society, Vol. 52 , N. 2. 
nerados por factores geográficos, como climas poco atractivos o sobrepoblación, y por factores normativos como leyes opresoras o altos impuestos. Sin embargo, afirmaba: "ninguna de estas causas es tan importante para explicar los volúmenes de inmigrantes como el deseo inherente de las personas de progresar en términos materiales".

Si bien la migración internacional obedece a múltiples y complejas causas, no es menos cierta la coincidencia con la apreciación de Ravenstein, al contrastarla con la realidad actual, en cuanto a las motivaciones que han detectado los estudiosos modernos, al responder que la principal causa es el $\boldsymbol{d e}$ seo de progreso material de los ciudadanos emigrantes.

En los últimos años, además del impacto que puede tener la migración en las personas y familias, se viene vinculando dicho fenómeno al desarrollo de los países, así encontramos un informe vigente de la Secretaría General de la Asamblea de Naciones Unidas, en cuyo resumen hace hincapié que "la migración internacional es un instrumento ideal para promover el desarrollo conjunto, esto es, la mejora coordinada o concertada de las condiciones económicas de los lugares de origen y de destino teniendo en cuenta la complementariedad entre ellos"s.

En dicho informe, se exponen muchos hechos relevantes, indicando, por ejemplo, que los fondos que los emigrantes envían a sus países en desarrollo (del que proceden) -por lo menos 167,000 millones de dólares solo en el 2005- superan con creces la suma de todas las modalidades de asistencia internacional.

Asimismo, se afirma que como consecuencia de la revolución de las comunicaciones y el transporte, los migrantes internacionales son hoy más que nunca, un dinámico eslabón humano entre culturas, economías y sociedades. Su riqueza no solo es cuantificable en dinero, sino que los conocimientos y las técnicas que acumulan son decisivos para la transferencia de tecnología, capital y memoria institucional en sus naciones.

Otros datos sobre migraciones internacionales refieren que en el año 2005, el número alcanzó los 191 millones de personas: de ellos, 115 millones vivían en países desarrollados y 75 millones en países en desarrollo. De todos ellos el $75 \%$ de migrantes se concentraban en solo 28 países: "Los Estados Unidos de América acogían a uno de cada cinco de todo el mundo" (Cuadro No 1 ).

Cuadro N.o 1. Países con el mayor número de migrantes: 1990 y 2005.

\begin{tabular}{|c|l|c|c|l|c|c|}
\hline \multirow{2}{*}{ Rango Pais } & \multicolumn{2}{|c|}{1990} & & \multicolumn{2}{c|}{ País } & \\
\cline { 3 - 4 } \cline { 6 - 7 } & & No Mig. MM & \% Total & & N0 Mig. MM & $\%$ Total \\
\hline 1 & EEUU & 23,3 & 15,0 & EEUU & 38,4 & 20,2 \\
2 & Fed. de Rusia & 11,5 & 7,4 & Fed. de Rusia & 12,1 & 6,4 \\
3 & India & 7,4 & 4,8 & Alemania & 10,1 & 5,3 \\
4 & Ucrania & 7,1 & 4,6 & Ucrania & 6,8 & 3,6 \\
5 & Pakistan & 6,6 & 4,2 & Francia & 6,5 & 3,4 \\
6 & Alemania & 5,9 & 3,8 & Arabia Saudita & 6,4 & 3,3 \\
7 & Francia & 5,9 & 3,8 & Canadá & 6,1 & 3,2 \\
8 & Arabia Saudita & 4,7 & 3,1 & India & 5,7 & 3,0 \\
9 & Canadá & 4,3 & 2,8 & Reino Unido & 5,4 & 2,8 \\
10 & Australia & 4,0 & 2,6 & España & 4,8 & 2,5 \\
11 & Irán & 3,8 & 2,5 & Australia & 4,1 & 2,2 \\
12 & Reino Unido & 3,8 & 2,4 & Pakistan & 3,3 & 1,7 \\
\hline
\end{tabular}

Fuente: Naciones Unidas, Trends in Total Migrant Stock:The 2005 Revisión / Elaboración Propia.

5 Asamblea General de las Naciones Unidas (2006) Sexagésimo Periodo de Sesiones, tema 54 c) del Programa Globalización e interdependencia: migración internacional y desarrollo. 
Es de destacar algunos cambios de posición producidos dentro de los países que soportan el mayor número de inmigrantes en los últimos años, siendo el más notable el ingreso de España (ver gráfico 2) como uno de los más atractivos destinos -ocupa el puesto 10 del ranking, con el $2.5 \%$ de los migrantes del planeta-, probablemente por el desarrollo económico experimentado en las tres últimas décadas.

Sobre el empleo mundial, la OIT señala que, aproximadamente, la mitad de todos los emigrantes integran la fuerza de trabajo mundial, es decir, 95 millones. Esta cifra incluye la totalidad de los migrantes que trabajan, independientemente de su admisión o condición jurídica.

\section{Efectos en América Latina}

En las notas publicadas por el Banco Interamericano de Desarrollo (BID), se señala que América Latina recibió más de US\$ $60 \mathrm{mil}$ millones de dólares de remesas en el 2006, cantidad que igualó a la inversión extranjera directa recibida ese año. Igualmente, el BID estima que para el 2010 las remesas a la región superaran los US\$ 100 mil millones de dólares, en contra de algunos vaticinios de analistas sobre un probable decrecimiento en dichos flujos.

Para el gerente del Fondo Multilateral de Inversiones del BID, Donald Terry, según comentario vertido al diario Correo de Lima, menciona que las actuales tendencias económicas y demográficas de América Latina y los países industrializados, hacen prever que las remesas aumentarán hasta llegar a US\$
110 millones anuales en el 2010. Sin embargo, explica que esto refleja la incapacidad de las economías de la región para brindarles mejores oportunidades de desarrollo a sus habitantes que terminan emigrando por las carencias en las que viven.

Esto hace intuir que el atractivo de obtener un trabajo bien remunerado en algún país rico sigue constituyéndose en el más poderoso incentivo para la migración internacional. Dicha atracción se intensifica a medida que aumentan las diferencias de ingreso entre países, no solo entre los países de ingresos altos y los de ingresos bajos, sino también se observa dentro de los países en desarrollo, entre los más pujantes y los menos dinámicos.

Sobre el efecto de la migración en países de América Latina, he encontrado trabajos muy interesantes que abogarían por el efecto benéfico que ella tiene en el desarrollo de algunos países, de los cuales resaltan:

- Sobre migración y desarrollo, J. Rocha ${ }^{6}$ afirma que existen indudables efectos positivos. Las migraciones en el país de origen pueden ser una válvula de escape para conflictos sociales, exportación del excedente de fuerza de trabajo, oportunidad de remesas, equilibrio de la balanza de pagos y transferencia tecnológica.

- En forma puntual, para el caso de El Salvador, pero cuya experiencia podría explicar lo que viene ocurriendo en países como el nuestro, Manuel Orozco ${ }^{7}$ señala que los viajes, las comunicaciones y el comercio de la nostalgia son áreas en las que los migrantes han adquirido un peso considerable en los últimos años, cita el caso del Grupo Taca-empresa de

6 Rocha, José Luis (2006). Población y desarrollo: Análisis de percepciones y aportes para una política de migraciones internacionales en Nicaragua. Acuerdo de Cooperación CELADE-UNFPA, Santiago de Chile - Naciones Unidas / CEPAL. 85 p. 22.

7 Orozco, Manuel (2005). “Transnacionalismo y desarrollo: tendencias y oportunidades en América Latina”. Foreing Affairs en español, Instituto Tecnológico Autónomo de México (ITAM), Vol. 5, N.o 3, Julio-Setiembre. 
transporte aéreo en América Centralvuela 21 veces al día de los Estados Unidos a El Salvador (por lo menos 70\% de sus clientes son centroamericanos), más de $60 \%$ de los migrantes centroamericanos llaman, por lo menos, una vez por semana y gastan al menos 30 minutos para hablar con parientes en el exterior (en total 120 minutos al mes), y los productos salvadoreños que consume la comunidad de El Salvador en la diáspora estadounidense representa al menos el $10 \%$ de las exportaciones totales de ese país a los EE.UU., o sea 450 millones de dólares. Según el estudio, "alimentos salvadoreños como harina para tortillas, frijol rojo, loroco, semita, queso y horchata son exportaciones importantes.

El estudio de Orozco también reveló que la mayoría de los emigrantes que visitan sus países permanece en ellos 15 días o más y gasta unos 65 dólares al día: "Visitar el país de origen entraña más que quedarse con la familia. Los inmigrantes que vuelven a casa son también turistas que gastan sumas considerables en diversión con sus familias, típicamente, al menos, 1000 dólares por estancia".

- En el tema de los beneficios que aporta la migración, el Profesor Teófilo Altamirano (Altamirano 2006: pp. 200 - 210) señala que "en los países de destino, los migrantes se convierten en líderes de la difusión de culturas nacionales...la migración transnacional hace "viajar" a las culturas y permite mostrar la variedad y la riqueza lingüística, religiosa, las grandes creaciones colectivas como la música, las danzas, la tradición oral, la comida, la artesanía...”.

En alusión al turismo, manifiesta que "las estadísticas oficiales en los países de ori- gen muestran que, aproximadamente, el $50 \%$ de turistas son los propios connacionales... (Estos) contribuyen al capital económico porque dejan en sus países de origen dólares que formarán partes de los ingresos del Estado y de la red de hoteles, agencias de turismo, restaurantes, artesanías, etc."

En lo concerniente a migración-inversión, comenta que... "existen muchos casos de migrantes laborales y profesionales que han retornado o están pensando retornar trayendo sus ahorros para invertir, en particular, en el turismo o en hotelería”.

\section{La migración nacional}

De la información oficial de la Dirección de Migraciones y Naturalización (DIGEMIN), podemos ver que el flujo migratorio neto de peruanos continua su avance, así el promedio de los 4 últimos años reportados supera los 405 mil peruanos que se van año a año, y si tomamos el promedio de migrantes del 2001 al 2004 frente al anterior, vemos que la migración ha crecido en $55 \%$ entre ambos periodos (ver cuadro 2).

Esta cifra tan significativa de compatriotas que decide quedarse en el extranjero, tiene una relación directa con las remesas que posteriormente ingresan al país y que en la actualidad, según diversos estimados superarían los 3.5 mil millones de dólares al año. Sobre la aplicación de las remesas, de acuerdo a los resultados de encuestas informados por el diario "El Comercio" en el 2008, sabemos que su efecto benéfico en las rentas familiares contribuye a hacer frente a los costos de alimentos, vivienda, educación y salud de la familia, mejorando de este modo el capital humano de esos hogares. 
Cuadro N.o 2. Flujos migratorios del Perú 2001-2008 (en miles)

\begin{tabular}{|c|c|c|c|c|}
\hline Años & Entradas & Salidas & $\underline{\text { Saldo Neto }}$ & Prom. \\
\hline 2008 & 1,365 & 1,753 & 388 & \\
\hline 2007 & 1,223 & 1,618 & 395 & 406 \\
\hline 2006 & 996 & 1,410 & 414 & \\
\hline 2005 & 908 & 1,333 & 425 & \\
\hline 2004 & 1,119 & 1,588 & 469 & \\
\hline 2003 & 710 & 942 & 232 & 262 \\
\hline 2002 & 734 & 959 & 225 & \\
\hline 2001 & 619 & 741 & 122 & \\
\hline
\end{tabular}

Gráfico N.o 1. Encuesta por tipo de gasto a partir de las remesas

CLASIFICACIÓN DE EMPRESAS POR SE

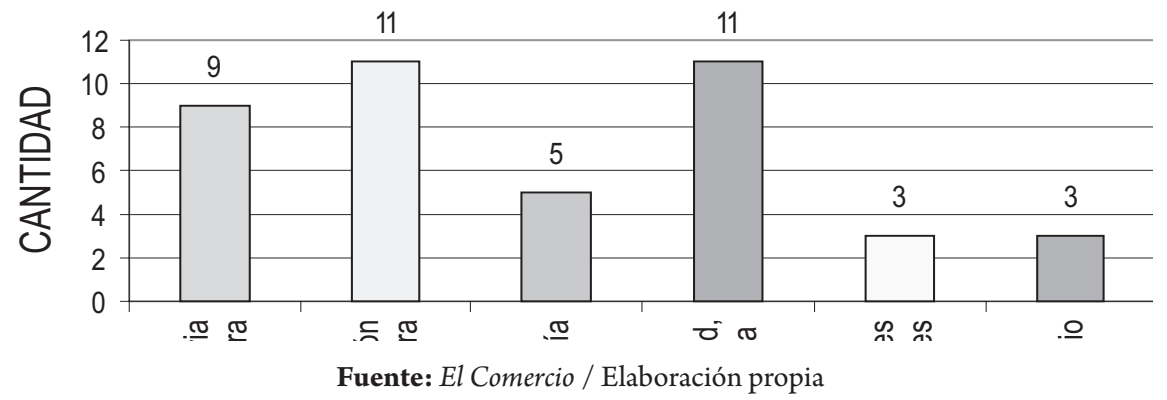

$\mathrm{Al}$ respecto, también el Instituto de Estadística e Informática (INEI) señalaba a los medios de prensa, en setiembre de 2008, que más de 400 mil hogares contaban al menos con un familiar que vivía en el extranjero y que les enviaban periódicamente dinero, ya sea para ahorrar y planear un mejor futuro $o$, en todo caso, como única fuente de ingreso para solventar todos los gastos de la casa.

Adicionalmente, podemos afirmar que los hogares que reciben remesas de los migrantes no solo tienen una mayor probabilidad de ahorrar, sino que además al gastar más ayudan a aumentar los ingresos en otros sectores de la economía. Por otro lado, la entrada de divisas asociadas a las remesas también me- jora la solvencia del país, colaborando en la disminución de los costos financieros (aunque no siempre se trasladan a los préstamos locales) y proporcionando el disponible para una financiación fiable en tiempos de inestabilidad.

Sobre su impacto en las actividades empresariales, podemos señalar que en números casos los empresarios migrantes hacen que aumente el comercio con su país de origen a través de la venta de productos elaborados en esos países, lo que, además, promueve y facilita el consumo propio de las colonias de peruanos asentadas en el extranjero, proveyéndolas de los bienes nacionales, que al considerar su número -hay más de 3 millones de 
peruanos en el extranjero- más su círculo de influencia, (parientes, amigos etc. de distinta nacionalidad), hacen que se conforme un segmento de mercado muy importante para los productos y servicios nacionales.
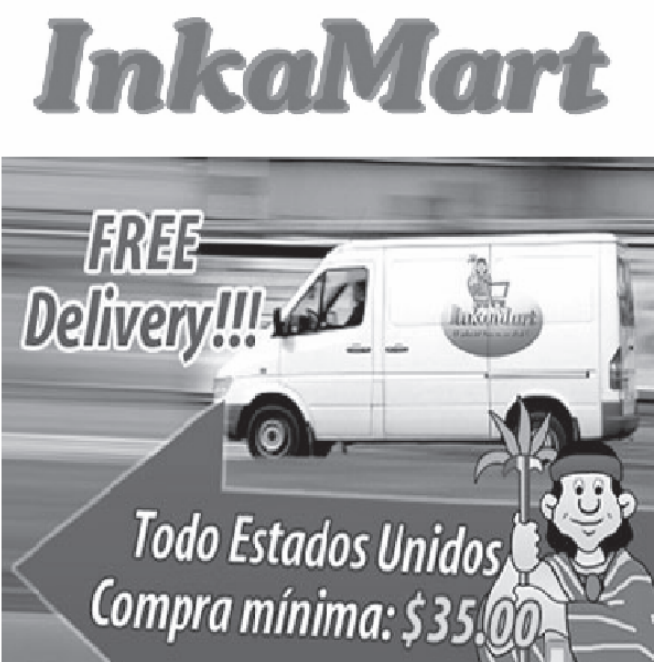

Tи оирентегсаdo ремиапо а las puertas de tu casa!!!!

(Empresa de distribución de migrante peruano en EE.UU.)
En este camino, expertos como Kloosterman y Rath ${ }^{8}$ señalan que está aumentando la actividad empresarial de los migrantes debido a transformaciones estructurales en las economías avanzadas, en que se está ampliando el sector de los servicios y también está incrementándose la producción en pequeña escala, que puede responder de manera flexible a los cambios de los gustos en los consumidores. Las empresas de propiedad de migrantes, con frecuencia, empiezan atendiendo a las necesidades de los personas de su misma etnia y solo más tarde diversifican sus actividades para servir al mercado más amplio.

$\mathrm{Al}$ observar las estadísticas oficiales de la SUNAD (Aduanas), notamos que en nuestro caso por ejemplo, dentro de las exportaciones más activas a los Estados Unidos, -el principal destino de los migrantes nacionales-, se encuentran las exportaciones no tradicionales y en estas, las partidas que se relacionan a productos y bebidas alimenticias vienen creciendo en forma notoria. Esto, se puede apreciar en el Gráfico anterior de

Gráfico N.o 2. Exportaciones peruanas de almentos hacia Estados Unidos.

Según volumen y valor FOB

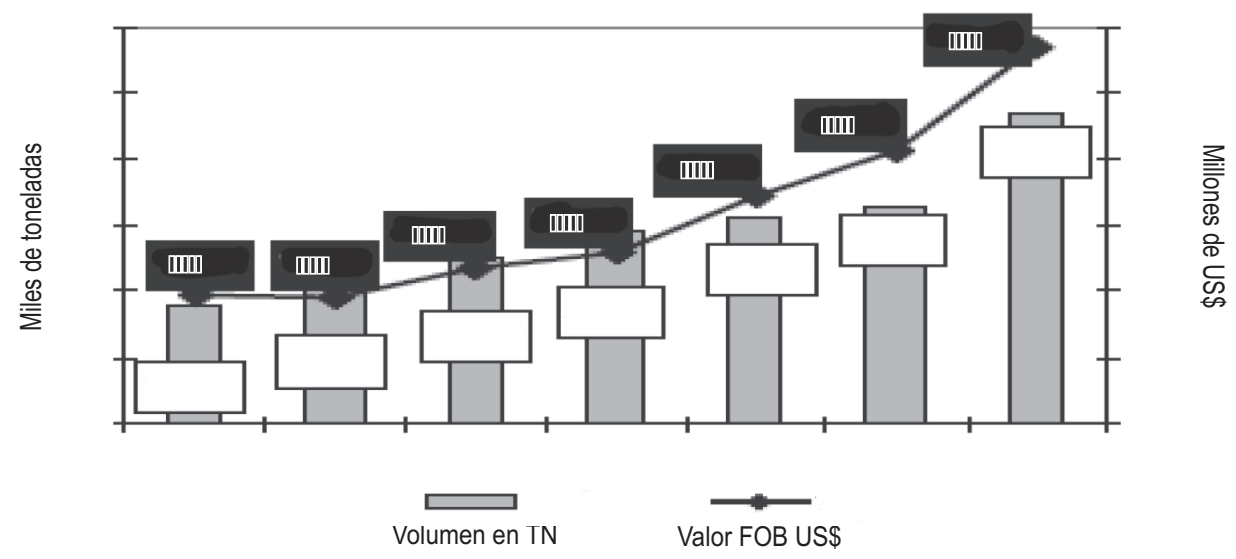

Fuente: ADUANAS.

Elaboración: Agro y agroindustriales - PROMPERU.

8 Kloosterman, Robert y Rath Jan (2003). Immigrant Entrepreneurs: Venturing Abroad in the Age of Globalization. Oxford y New York, Berg. 
PROMPERÚ, donde de \$ 193.5 millones de dólares exportados en el año 2000 se pasa a \$ 589.1 millones de dólares en el año 2006, equivalente a un crecimiento del $304 \%$ en el periodo analizado.

Relacionando a las cifras anteriores de incremento exportador, también debemos resaltar la fama y el auge que está adquiriendo la cocina peruana internacionalmente, la cual a través de la conquista de mercados con restaurantes en numerosos países, está actuando como una palanca virtuosa en la promoción del consumo de alimentos y bebidas peruanas en el mundo. Consumo que comprende variados productos e insumos de origen nacional -productos étnicos-, presentados en diversas formas e importados y distribuidos por empresas residentes en el extranjero, algunas de fuerte vinculación con nuestros migrantes nacionales.

\section{CONCLUSIONES}

1. Un factor fundamental para el crecimiento de las exportaciones a los EE.UU., sobre todo relacionadas a productos "no tradicionales", lo constituye el creciente consumo de los connacionales y sus círculos de allegados residentes en dicho país, Este mayor consumo directo se viene dando en lugares públicos como restaurantes y en el ambiente privado familiar.

2. Existe presumiblemente un amplio mercado potencial en el extranjero, conformado por el segmento cuya base integran los residentes peruanos en el extranjero y sus círculos de influencia (familiares y amigos no nacionales), los cuales podrían impulsar la producción de la industria de bienes y servicios del país.

3. Un hecho favorable que está contribuyendo en la demanda de productos e ingredientes alimenticios peruanos es la promoción de su comida por emprendedores de negocios. Algunos, aprovechando mercados con preferencia por los platos peruanos, han inaugurado restaurantes en países de fuerte migración nacional como los Estados Unidos, España, Chile etc., beneficiando con esto las exportaciones de más insumos y productos nacionales, incluyendo las bebidas tradicionales y espirituosas del Perú.

\section{RECOMENDACIONES}

Las empresas vinculadas a la producción de bienes especialmente de necesidades primarias (alimentación y vestido), deben incorporar en sus planes empresariales de crecimiento al segmento de residentes peruanos y sus círculos de allegados en los países con mayor número de migrantes peruanos, como parte del mercado potencial a ser atendido.

El Estado peruano debe considerar en sus políticas de desarrollo sectorial -industria, comercio exterior, turismo- al mercado constituido por las numerosas colonias de peruanos en el extranjero, cuyos integrantes pueden tener una capacidad de compra superior a la media de los consumidores de nuestro país. En tal sentido, por ejemplo, las campañas de difusión y propaganda que transmita el gobierno peruano para incentivar el consumo de bienes nacionales deben ser acondicionadas para los peruanos migrantes.

Los gobiernos e instituciones privadas y públicas regionales en el país deben procurar, mantener y estrechar los vínculos existentes con la población que ha emigrado desde sus pueblos al extranjero, ya que de esta forma se fortalecerá y se hará más efectiva su colaboración con el proceso de crecimiento de las exportaciones y el turismo regional, y por ende, en el desarrollo del Perú. 


\section{REFERENCIAS BIBLIOGRÁFICAS}

1. Altamirano, Teófilo (2000). Liderazgo $y$ organizaciones de peruanos en el exterior. Culturas transnacionales e imaginario sobre el desarrollo. PROMPERU, Fondo Editorial de la PUCP.

2. Altamirano, Teofilo (2006). Remesas y nueva "fuga de cerebros". Impactos transnacionales. Pontificia Universidad Católica del Perú. Fondo Editorial. $246 \mathrm{pp}$.

3. Aquino, Carlos (1999). "Migración internacional del trabajo: el caso de los peruanos en Japón".

4. BID - Fondo Multilateral de Inversiones (2005). Enviando dinero a casa: Las remesas como herramienta de desarrollo en América Latina y El Caribe. Santiago, Chile. Web: www.iadb.org

5. De los Ríos, Juan / Rueda, Carlos (2005). ¿Por qué migran los peruanos al exterior? Fondo Concursable de Proyectos de Investigación (FOCPI). V.P. Marzo. Doc. $45 \mathrm{pp}$.
6. Kloosterman, Robert y Rath Jan (2003). Immigrant entrepreneurs: Venturing abroad in the age of globalization, Oxford y New York.

7. Massey, D., Arango, J., Hugo, G., Kouaouci, A., Pellegrino, A., Edward Taylor, J. (1993). Theories of international migration: A review and appraisal. Population and development review. Vol. 19, N. 3, 431-466, September.

8. Naciones Unidas (2006). Globalización e interdependencia: migración internacional y desarrollo. Asamblea General, Tema 54 c) del programa, 97 pp.

9. OIM (2003). "World migration 2003. Managing migration challenges and responses for people on the move". Vol. 2 - OIM World Migration Report Series, Genova: OIM.

10. Rocha Gómez, José Luis (2006). “Análisis de percepciones y aportes para una política de migraciones internacionales en Nicaragua”. CELADE. Santiago de Chile. Doc. 85 pp. 